\title{
Role of per-rectal portal scintigraphy in long-term follow-up of congenital portosystemic shunt
}

\author{
Yuki Cho', Daisuke Tokuhara', Taro Shimono², Akira Yamamoto², Shigeaki Higashiyama ${ }^{3}$, Kohei Kotani ${ }^{3}$, Joji Kawabe ,
} Yoshiyuki Okano ${ }^{4}$, Susumu Shiomi ${ }^{3}$ and Haruo Shintaku'

BACKGROUND: Congenital portosystemic shunt (CPSS) has the potential to cause hepatic encephalopathy and thus needs long-term follow-up, but an effective follow-up method has not yet been established. We aimed to evaluate the importance of per-rectal portal scintigraphy (PRPS) for long-term follow-up of CPSS.

METHODS: We retrospectively examined shunt severity time course in patients (median: 9.6 y, range: 5.2-16.6 y) with intrahepatic $(n=3)$ or extrahepatic $(n=3)$ CPSS by using blood tests, ultrasonography or computed tomography, and PRPS. Per-rectal portal shunt index (cutoff: 10\%) was calculated by PRPS.

RESULTS: PRPS demonstrated that the initial shunt index was reduced in all intrahepatic cases (from $39.7 \pm 9.8 \%$ (mean \pm SD) to $14.6 \pm 4.7 \%$ ) and all extrahepatic cases (from $46.2 \pm 10.9$ to $27.5 \pm 12.6 \%)$ during the follow-up period. However, ultrasonography and computed tomography disclosed different shunt diameter time courses between intrahepatic and extrahepatic CPSSs. Initial shunt diameter $(5.8 \pm 3.5 \mathrm{~mm})$ reduced to $2.0 \pm 0.3 \mathrm{~mm}$ in intrahepatic cases, but the initial diameter $(6.3 \pm 0.7 \mathrm{~mm})$ increased to $10.6 \pm 1.0 \mathrm{~mm}$ in extrahepatic cases. All patients had elevated serum total bile acid or ammonia levels at initial screening, but these blood parameters were insufficient to assess shunt severity because the values fluctuate. CONCLUSION: PRPS can track changes in the shunt severity of CPSS and is more reliable than ultrasonography and computed tomography in patients with extrahepatic CPSS.

C ongenital portosystemic shunt (CPSS), which is a major cause of neonatal hypergalactosemia without galactosemetabolizing-enzyme deficiency (1), causes brain manganese deposition, pulmonary hypertension, and hyperammonemia leading to hepatic encephalopathy (2-8). CPSS is generally suspected if serum total bile acid (TBA) and ammonia levels are elevated, and it is diagnosed by using color Doppler ultrasonography, dynamic contrast-enhanced computed tomography (CT), and per-rectal portal scintigraphy (PRPS) $(7,9-12)$. Some shunts close spontaneously, whereas others need to be closed surgically or with embolization because of hyperammonemia leading to severe hepatic encephalopathy $(7,8,13-15)$. Therefore, it is important to follow up CPSS patients carefully with color Doppler ultrasonography, dynamic contrast-enhanced CT, and blood tests. Despite this, there is no gold standard for accurately assessing the degree of shunt. Color Doppler ultrasonography and dynamic contrastenhanced CT are useful for detecting shunt location and for assessing shunt diameter and flow, but these imaging modalities cannot be used to evaluate shunt severity quantitatively. By contrast, PRPS can be used to calculate a shunt index (SI) for quantifying shunt severity, as previously reported (9). Currently, the use of PRPS is limited to the diagnosis of CPSS, and its application to long-term follow-up of CPSS is uncertain. Here, we aimed to clarify the role for PRPS in the long-term follow-up of patients with CPSS by retrospectively evaluating changes in shunt severity over time as assessed with PRPS.

\section{RESULTS}

\section{Patients}

Six patients (mean age: $9.6 \mathrm{y}$; range: $5.2-16.6$ y) were diagnosed as having CPSS during the first year of life. On the basis of ultrasonography and dynamic contrast-enhanced CT, three of the six patients were identified as having intrahepatic CPSS, and the remaining three patients were found to have extrahepatic CPSS (Table 1). None of the six patients had abnormalities in the abdominal cavity, including hepatic tumors. Two of the patients with intrahepatic CPSS had shunts between the left portal vein and the central hepatic vein, and one had a shunt between the left portal vein and left hepatic vein (Table 1). Of the patients with extrahepatic CPSS, two had splenorenal shunts (Table 1; Figure 1a,b), and one had a mesocaval shunt (Table 1; Figure 1c; Supplementary Video S1 online). All six patients had normal mental development without hepatic encephalopathy and showed absence of pulmonary hypertension.

\section{Shunt Diameter}

Ultrasonography or dynamic contrast-enhanced CT, or both, disclosed a difference between intrahepatic and extrahepatic CPSSs in terms of changes in shunt diameter over time. All three 
Table 1. Profiles of six children with congenital portosystemic shunt

\begin{tabular}{|c|c|c|c|c|c|c|c|c|}
\hline \multirow[b]{2}{*}{ Patient no. } & \multirow{2}{*}{$\begin{array}{l}\text { Age at initial } \\
\text { examination } \\
\text { (mo) }\end{array}$} & \multirow[b]{2}{*}{ Sex } & \multirow[b]{2}{*}{ Shunt location } & \multicolumn{5}{|c|}{ Initial laboratory findings } \\
\hline & & & & $\begin{array}{l}\text { Galactose }^{\mathrm{a}} \\
(<8 \mathrm{mg} / \mathrm{dl})\end{array}$ & $\begin{array}{c}\text { TBA } \\
(<10 \mu \mathrm{mol} / \mathrm{l})\end{array}$ & $\begin{array}{c}\mathrm{NH}_{3} \\
(30-80 \mu \mathrm{g} / \mathrm{dl})\end{array}$ & $\begin{array}{c}\text { AST } \\
(20-70 \mathrm{IU} / \mathrm{I})\end{array}$ & $\begin{array}{c}\text { ALT } \\
(10-70 \mathrm{IU} / \mathrm{l})\end{array}$ \\
\hline 2 & 1 & Male & Intrahepatic (LPV-CHV) & 8.9 & 44 & 89 & 41 & 28 \\
\hline 3 & 1 & Male & Intrahepatic (LPV-CHV) & 11.6 & 56 & 141 & 30 & 19 \\
\hline 6 & 1 & Male & Extrahepatic (IMV-IIV) & 7.4 & 58 & 80 & 38 & 23 \\
\hline
\end{tabular}

ALT, alanine aminotransferase; AST, aspartate aminotransferase; CHV, central hepatic vein; IIV, internal iliac vein; IMV, inferior mesenteric vein; IU, international units; LHV, left hepatic vein; LPV, left portal vein; LRV, left renal vein; SV, splenic vein; TBA, total bile acid.

a Galactose was evaluated within $45 \mathrm{~d}$ after birth by newborn mass screening.

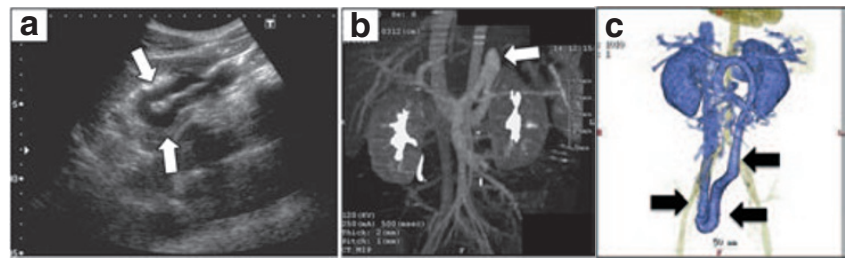

Figure 1. Ultrasonography and computed tomography (CT) portal venography of extrahepatic congenital portosystemic shunt patients. (a) Splenorenal shunt (arrow) was clearly visualized in the sagittal view on ultrasonography (16y). (b) Splenorenal shunt (arrow) on maximum intensity projection image of CT portal venography (1.5 y). (c) Mesocaval shunt (arrow) on three-dimensional volume-rendered image of CT portal venography $(5 \mathrm{y})$.

intrahepatic CPSS patients showed spontaneous shunt regression. The initial shunt diameter of $5.8 \pm 3.5 \mathrm{~mm}$ (mean \pm SD) at $0.7 \pm 0.5 \mathrm{y}$ of age was reduced to $2.0 \pm 0.3 \mathrm{~mm}$ at $6 \pm 2.6 \mathrm{y}$ of age $-\mathrm{a}$ reduction of $59.6 \pm 16.3 \%$ over $5.2 \pm 2.8$ y (Figure $2 a-c$ ). By contrast, the shunt diameter increased in all three extrahepatic CPSS patients: the initial shunt diameter of $6.3 \pm 0.7 \mathrm{~mm}$ at $0.6 \pm 0.2 \mathrm{y}$ of age increased to $10.6 \pm 1.0 \mathrm{~mm}$ at $11.3 \pm 5.6 \mathrm{y}$ of age-an increase of $70.0 \pm 25.8 \%$ over $10.6 \pm 5.6$ y (Figure $2 d-f$ ).

\section{Shunt Index}

PRPS was performed in each patient two to four times at a median interval of $4.5 \mathrm{y}$ (range: 1.4-6.7 y). For all six patients, the SI derived from PRPS decreased from $42.9 \pm 9.9 \%$ (mean \pm SD, cutoff: $10 \%$ ) at $0.9 \pm 0.8 \mathrm{y}$ of age to $21.1 \pm 11.1 \%$ at $9.3 \pm 4.9$ $\mathrm{y}$ of age (Figure 2; Supplementary Figure S1 online)-a decrease of $51.8 \pm 18.9 \%$ over $8.3 \pm 5.2 \mathrm{y}$. In the patients with intrahepatic CPSS, the initial SI of $39.7 \pm 9.8 \%$ at $1.2 \pm 1.1 \mathrm{y}$ of age decreased to $14.6 \pm 4.7 \%$ at $6.6 \pm 2.6 \mathrm{y}$ of age (Figure 2a-c; Supplementary Figure S1 online) - a reduction of $60.7 \pm 19.9 \%$ over $5.4 \pm 3.1$ y. In the patients with extrahepatic CPSS, the initial SI of $46.2 \pm 10.9 \%$ at $0.6 \pm 0.4 \mathrm{y}$ of age decreased to $27.5 \pm 12.6 \%$ at $11.8 \pm 5.6 \mathrm{y}$ of age (Figure 2d-f; Supplementary Figure S1 online)-a reduction of $42.9 \pm 16.3 \%$ over $11.3 \pm 5.7 \mathrm{y}$.

\section{Blood Tests}

Hypergalactosemia was identified in all six patients by newborn screening (Table 1). At the initial examination at our hospital, serum levels of TBA or ammonia were elevated in all six patients (Table 1). No elevations of aspartate aminotransferase and alanine aminotransferase levels were found in any of these patients. During the follow-up period, galactose levels decreased to within the normal range by 2 y of age despite persistent elevation of TBA and ammonia levels (Figure 3). The persistently high values of TBA or ammonia suggested the presence of a shunt, but the values fluctuated because of intestinal motility and changes in the types of meals consumed before fasting, limiting the use of these blood parameters in the quantitative assessment of shunt severity.

\section{DISCUSSION}

We retrospectively evaluated the long-term clinical course of CPSS patients, focusing on the degree of shunt as determined with PRPS. Various imaging modalities and blood tests indicated that the natural histories of intrahepatic and extrahepatic CPSSs differed. Intrahepatic CPSS without hepatic tumor has been reported to spontaneously close or regress, whereas extrahepatic CPSS does not spontaneously regress $(3,7,8,16-18)$. Ultrasonography and dynamic contrast-enhanced CT evaluation showed spontaneous reductions in the shunt size in all of our patients with intrahepatic CPSS but in none with extrahepatic CPSS, strengthening the previous findings. Furthermore, all of our patients with extrahepatic CPSS showed marked enlargement in shunt diameter over time. The reason for this difference between intrahepatic and extrahepatic CPSSs is unclear; however, one possible explanation is the difference in the environments surrounding intrahepatic and extrahepatic CPSSs. An intrahepatic CPSS is tightly surrounded by liver parenchyma and may be under pressure during growth, perhaps leading to spontaneous closure. One case report of a child with intrahepatic CPSS who died of pulmonary hypertension described an enlarged portal tract with multiple thin-walled angiomatous vessels; these may be a feature of shunts that tend to close or regress under pressure (5). By contrast, an extrahepatic CPSS is under less pressure from the surrounding tissues and thus may retain its size, or enlarge, as the patient grows.

The most important finding of our study is the reduction in SI in extrahepatic CPSS, despite the increase in shunt diameter. PRPS is a noninvasive method that results in little exposure to 
a

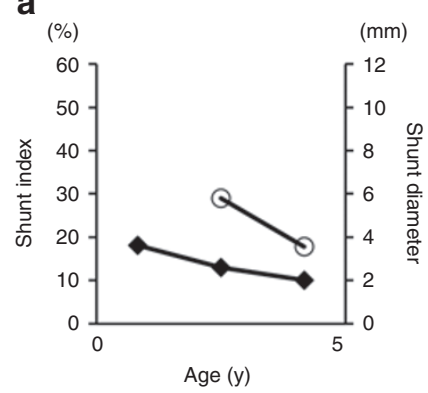

d

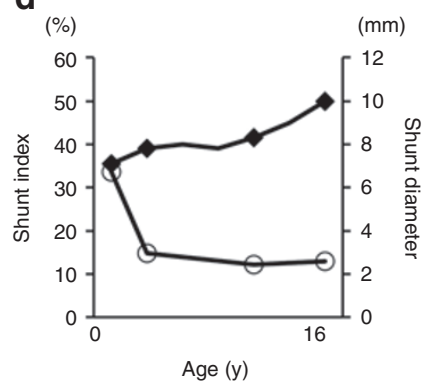

b

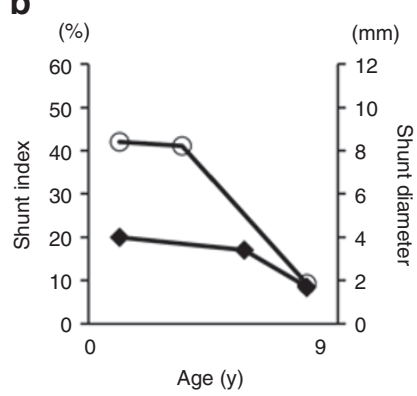

e

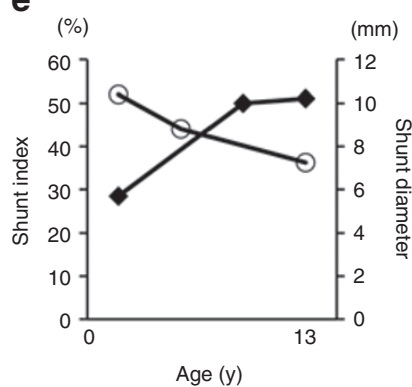

C

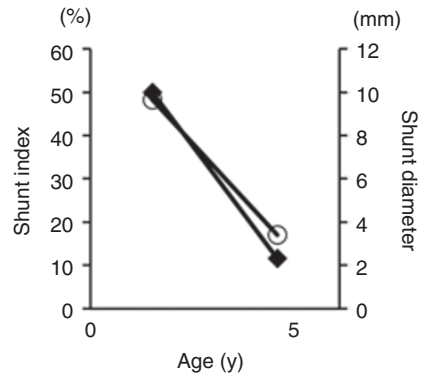

f

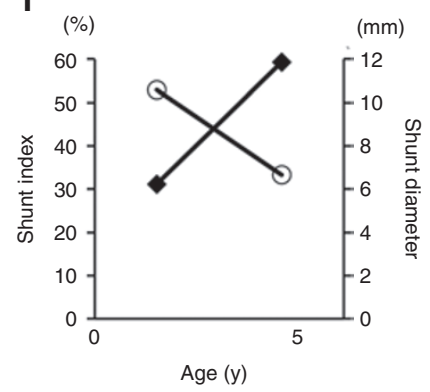

Figure 2. Time course of shunt index and shunt diameter in patients with congenital portosystemic shunt (CPSS). (a) Case 1 with intrahepatic CPSS between LPV and LHV. (b) Case 2 with intrahepatic CPSS between LPV and CHV. (c) Case 3 with intrahepatic CPSS between LPV and CHV. (d) Case 4 with extrahepatic CPSS between SV and LRV. (e) Case 5 with extrahepatic CPSS between SV and LRV. (f) Case 6 with extrahepatic CPSS between IMV and IIV. Case number is same as the patient number. Shunt index is shown as open circle, and shunt diameter is shown as solid diamond. CHV, central hepatic vein; IMV, inferior mesenteric vein; IIV, internal iliac vein; LHV, left hepatic vein; LPV, left portal vein; LRV, left renal vein; SV, splenic vein.

radionuclide and is an effective method for evaluating portal circulation; it is therefore used to diagnose or evaluate CPSS in children and to assess the severity of cirrhosis in adults $(9,19-21)$. Shiomi et al. (19) reported the usefulness of PRPS in providing detailed information about changes in portal hemodynamics; they reported that SI in adults increases as liver cirrhosis progresses. Uchino et al. (3) reported that the risk of hepatic encephalopathy increases with the degree of portosystemic shunting, as indicated by the PRPS SI. The SI has also been shown to be useful for evaluating the postoperative course in dogs with extrahepatic CPSS (22). In children, a recent study demonstrated that PRPS is complementary to ultrasonography and endoscopy in the assessment of portal hypertension associated with chronic cholestasis (23). We found that SI decreased in all of our subjects with CPSS during long-term follow-up; this may be a previously unrecognized feature of the natural course of CPSS in humans. However, the change in SI paralleled a reduction in shunt diameter in the children with intrahepatic CPSS but contrasted with the increase in shunt diameter in patients with extrahepatic CPSS. CPSS without spontaneous closure or regression is considered to reflect an increase in the degree of shunt severity and is associated with complications (16). In addition, the enlargement of shunt diameter in our patients with extrahepatic CPSS may have led physicians to consider that the severity of the shunt has worsened. However, our study demonstrated that an increase in shunt diameter, as shown by imaging modalities such as ultrasonography and dynamic contrast-enhanced CT, is not an indicator of the severity of the shunt in CPSS, whereas PRPS can be used to quantify shunt severity by using the SI, regardless of changes in shunt appearance.
Imaging modalities such as ultrasonography and dynamic contrast-enhanced CT are useful for detecting the location and size of CPSS but cannot provide the degree of shunt severity as quantitatively as can PRPS. In addition, the image quality of ultrasonography for extrahepatic CPSS is often influenced by abdominal conditions (e.g., intestinal contents) because the shunt is surrounded by the stomach or the small or large intestine, whereas PRPS is not influenced by abdominal conditions because the radiological agent is instilled through the rectum. The concentrations of TBA and ammonia are useful for monitoring the presence of PSS, but because these values can fluctuate with changes in gut conditions, their use in accurately assessing progress toward shunt closure is problematic. Therefore, it is important and effective to use PRPS in addition to ultrasonography, dynamic contrast-enhanced CT, and blood tests to assess shunt severity in the diagnosis and followup of CPSS.

Surgical repair or embolization may be recommended for extrahepatic CPSS without closure because of the high risk of hepatopulmonary syndrome, pulmonary hypertension, and hepatic encephalopathy (4,7,8,13-16). Stringer (8) described that CPSS-affected individuals are at risk of developing hepatic encephalopathy and/or an intrahepatic tumor depending largely on the volume and duration of the shunt. The risk of hepatic encephalopathy is related to the degree of portosystemic shunting, as measured by PRPS (3); therefore, it is rational to take the SI into consideration when deciding whether surgical treatment or embolization is indicated. On the other hand, Bernard et al. (7) recommended that, even when no complication is detected, closure of shunt should be considered early to prevent complications 
a
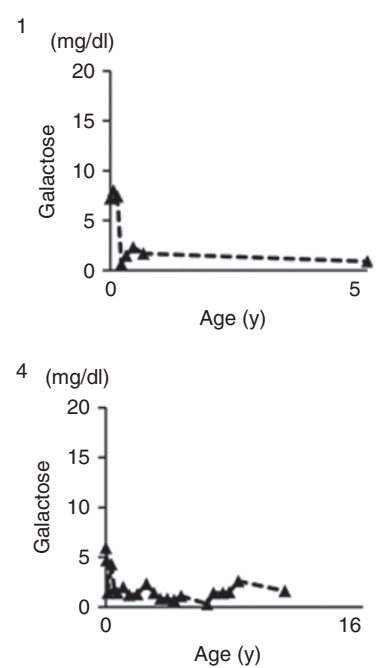

b

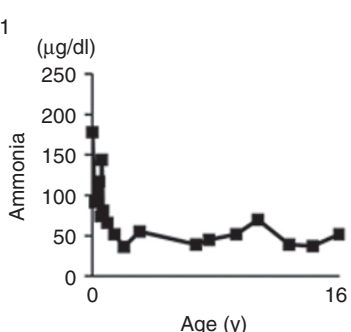

4

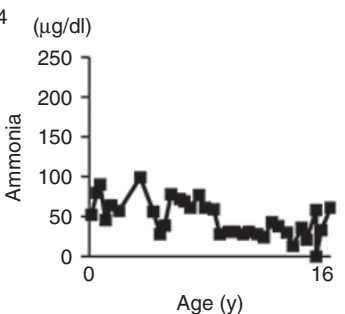

C
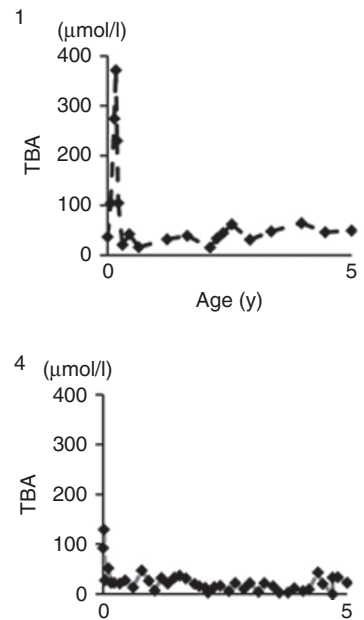

Age (y)
2

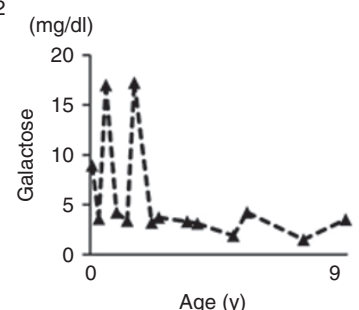

5 (mg/dl)

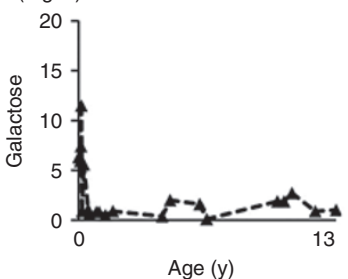

2

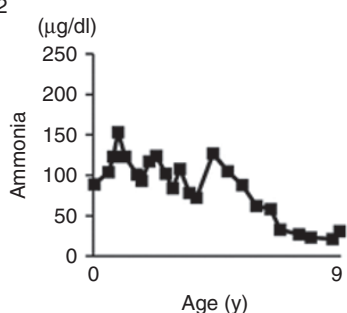

5

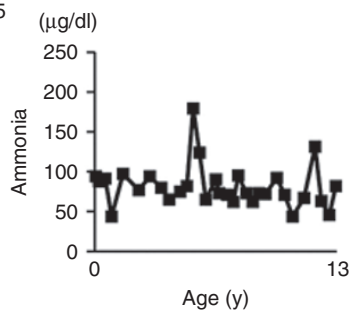

2

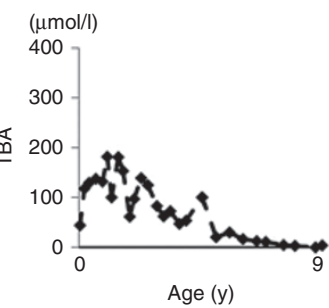

5

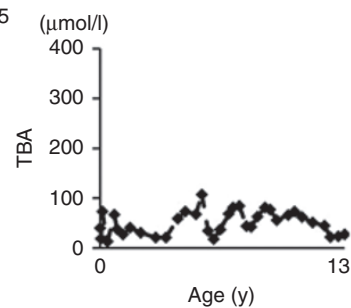

$3(\mathrm{mg} / \mathrm{dl})$

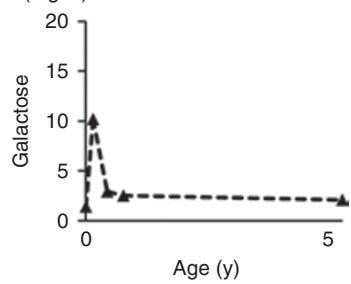

$6 \quad(\mathrm{mg} / \mathrm{dl})$
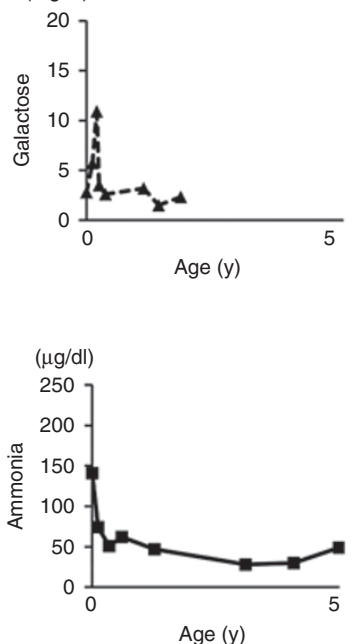

6
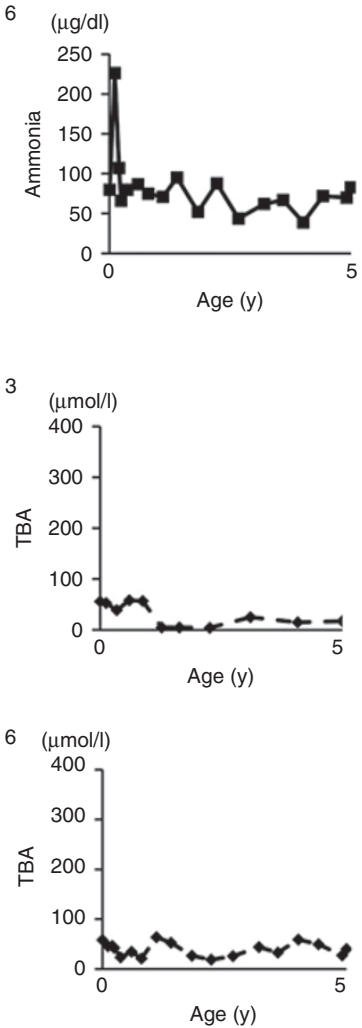

Figure 3. Time course of (a) galactose, (b) TBA, and (c) ammonia levels in children with congenital portosystemic shunt (CPSS). (1) Case 1 with intrahepatic CPSS between LPV and LHV. (2) Case 2 with intrahepatic CPSS between LPV and CHV. (3) Case 3 with intrahepatic CPSS between LPV and CHV. (4) Case 4 with extrahepatic CPSS between SV and LRV. (5) Case 5 with extrahepatic CPSS between SV and LRV. (6) Case 6 with extrahepatic CPSS between IMV and IIV. Case number is the same as patient number. CHV, central hepatic vein; IIV, internal iliac vein; IMV, inferior mesenteric vein; LHV, left hepatic vein; LPV, left portal vein; LRV, left renal vein; SV, splenic vein; TBA, total bile acid. 
in cases of CPSS except for small intrahepatic shunt. Therefore, it remains unclear whether SI affects the indications for closure of shunt and there are no criteria for initiating treatment based on PRPS. In our study, the SI of extrahepatic CPSS decreased even though the shunt diameter increased during the long-term natural course of the condition. Taking the current and previous findings together suggests that it is important to determine the SI to evaluate the risk of hepatic encephalopathy in patients with extrahepatic CPSS; in addition, SI may be considered an important parameter in addition to hyperammonemia, portal pressure, shunt size, and clinical symptoms suggesting hepatic encephalopathy when deciding whether surgical treatment or embolization is warranted in patients with extrahepatic CPSS, although further clinical study is necessary to support this hypothesis.

In conclusion, our study clearly revealed that PRPS can provide information about the severity of CPSS beyond that provided by biochemical tests, ultrasonography, and dynamic contrast-enhanced CT. SI decreased over time, both in patients with intrahepatic CPSS, whose shunts decreased in diameter, and in patients with extrahepatic CPSS, whose shunts increased in diameter. We recommend following up patients with CPSS by using PRPS.

\section{METHODS}

Six patients with CPSS were retrospectively analyzed. Informed consent was obtained, and the study was approved by the Institutional Review Board of Osaka City University Hospital. All six patients were found to have hypergalactosemia during newborn screening but did not have galactose-metabolizing-enzyme deficiency. Each patient was diagnosed as having CPSS by blood tests, including aspartate aminotransferase, alanine aminotransferase, galactose, TBA, and ammonia levels, in addition to ultrasonography, dynamic contrast-enhanced CT, and PRPS. All patients were followed using the same modalities. Shunt location, shunt flow, and shunt diameter were evaluated by using ultrasonography and dynamic contrast-enhanced CT (Table 1). Chest roentgenography, electrocardiography, and echocardiography were undertaken to assess pulmonary hypertension. PRPS was performed as previously described (9). Briefly, a polyethylene tube (Nélaton's catheter, French 8-12, Terumo Cooperation, Tokyo, Japan) was inserted $10 \mathrm{~cm}$ deep into the rectum, reaching the upper part. A large-field scintillation camera (Vertex Plus; Adac Laboratories, Milpitas, CA) was used to generate time-activity curves. The camera had a low-energy, multipurpose, parallel-hole collimator and was interfaced with a digital computer (Pegasys; Adac Laboratories). The camera was positioned over the patient's abdomen so that the field of view included the heart, liver, and spleen. First, $111 \mathrm{MBq}$ (megabecquerels) of Tc-99m-pertechnetate $(1 \mathrm{ml})$ was given through the tube, followed by $10-20 \mathrm{ml}$ of air. Thereafter, time-activity curves for the areas of the liver and heart were obtained every $4 \mathrm{~s}$. At the end of the 5 -min examination, the 5-min summed image, displayed in color, was recorded. To evaluate the extent of the portosystemic shunt in terms of an SI, we calculated the ratio of counts for the liver to counts for the heart integrated for $24 \mathrm{~s}$ immediately after the appearance of the liver time-activity curve.

\section{SUPPLEMENTARY MATERIAL}

Supplementary material is linked to the online version of the paper at http://www.nature.com/pr

\section{REFERENCES}

1. Nishimura Y, Tajima G, Dwi Bahagia A, et al. Differential diagnosis of neonatal mild hypergalactosaemia detected by mass screening: clinical significance of portal vein imaging. J Inherit Metab Dis 2004;27:11-8.
2. Mizoguchi N, Nishimura Y, Ono H, Sakura N. Manganese elevations in blood of children with congenital portosystemic shunts. Eur J Pediatr 2001;160:247-50.

3. Uchino T, Matsuda I, Endo F. The long-term prognosis of congenital portosystemic venous shunt. J Pediatr 1999;135(2 Pt 1):254-6.

4. Eroglu Y, Donaldson J, Sorensen LG, et al. Improved neurocognitive function after radiologic closure of congenital portosystemic shunts. J Pediatr Gastroenterol Nutr 2004;39:410-7.

5. Ersch J, Bänziger O, Braegger C, Arbenz U, Stallmach T. An infant with pulmonary hypertension due to a congenital porto-caval shunt. Eur J Pediatr 2002;161:660-2.

6. Ohno T, Muneuchi J, Ihara K, et al. Pulmonary hypertension in patients with congenital portosystemic venous shunt: a previously unrecognized association. Pediatrics 2008;121:e892-9.

7. Bernard O, Franchi-Abella S, Branchereau S, Pariente D, Gauthier F, Jacquemin E. Congenital portosystemic shunts in children: recognition, evaluation, and management. Semin Liver Dis 2012;32:273-87.

8. Stringer MD. The clinical anatomy of congenital portosystemic venous shunts. Clin Anat 2008;21:147-57.

9. Shiomi S, Sasaki N, Ikeoka N, et al. Usefulness of per-rectal portal scintigraphy with Tc-99m pertechnetate for galactosemia in infants. Ann Nucl Med 1998;12:375-8.

10. Kono T, Hiki T, Kuwashima S, Hashimoto T, Kaji Y. Hypergalactosemia in early infancy: diagnostic strategy with an emphasis on imaging. Pediatr Int 2009;51:276-82.

11. Mizoguchi N, Sakura N, Ono H, Naito K, Hamakawa M. Congenital portoleft renal venous shunt as a cause of galactosaemia. J Inherit Metab Dis 2001;24:72-8

12. Sakura N, Mizoguchi N, Eguchi T, et al. Elevated plasma bile acids in hypergalactosaemic neonates: a diagnostic clue to portosystemic shunts. Eur J Pediatr 1997;156:716-8.

13. Yamagami T, Yoshimatsu R, Matsumoto T, et al. Successful embolization using interlocking detachable coils for a congenital extrahepatic portosystemic venous shunt in a child. J Pediatr Surg 2007;42: 1949-52.

14. Ikeda S, Sera Y, Ohshiro H, Uchino S, Uchino T, Endo F. Surgical indications for patients with hyperammonemia. J Pediatr Surg 1999;34: 1012-5.

15. Kimura T, Soh H, Hasegawa T, et al. Laparoscopic correction of congenital portosystemic shunt in children. Surg Laparosc Endosc Percutan Tech 2004; 14:285-8.

16. Franchi-Abella S, Branchereau S, Lambert V, et al. Complications of congenital portosystemic shunts in children: therapeutic options and outcomes. J Pediatr Gastroenterol Nutr 2010;51:322-30.

17. Gitzelmann R, Forster I, Willi UV. Hypergalactosaemia in a newborn: self-limiting intrahepatic portosystemic venous shunt. Eur J Pediatr 1997;156:719-22.

18. Velayutham P, Dev A, Arora A. Congenital extrahepatic portocaval shunt: growth in vain. J Pediatr 2013;162:1076.e1.

19. Shiomi S, Kuroki T, Kurai O, et al. Portal circulation by technetium99m pertechnetate per-rectal portal scintigraphy. J Nucl Med 1988;29: 460-5.

20. Yoshimoto Y, Shimizu R, Saeki T, et al. Patent ductus venosus in children: a case report and review of the literature. J Pediatr Surg 2004;39: E1-5.

21. Kawamura E, Habu D, Hayashi T, et al. Natural history of major complications in hepatitis $\mathrm{C}$ virus-related cirrhosis evaluated by per-rectal portal scintigraphy. World J Gastroenterol 2005;11:3882-6.

22. Van Vechten BJ, Komtebedde J, Koblik PD. Use of transcolonic portal scintigraphy to monitor blood flow and progressive postoperative attenuation of partially ligated single extrahepatic portosystemic shunts in dogs. J Am Vet Med Assoc 1994;204:1770-4.

23. Vajro P, Celentano L, Manguso F, et al. Per-rectal portal scintigraphy is complementary to ultrasonography and endoscopy in the assessment of portal hypertension in children with chronic cholestasis. J Nucl Med 2004;45:1705-11. 